\title{
Ultrasound-guided supraclavicular brachial plexus block in pediatric patients -A report of four cases-
}

\author{
Chun Woo Yang ${ }^{1}$, Choon-Kyu Cho ${ }^{1}$, Hee Uk Kwon ${ }^{1}$, Jae Young Roh ${ }^{2}$, Youn Moo $\mathrm{Heo}^{2}$, and \\ Sung-Min $\mathrm{Ahn}^{3}$ \\ Departments of ${ }^{1}$ Anesthesiology and Pain Medicine, ${ }^{2}$ Orthopedic Medicine, College of Medicine, Konyang University, Daejeon, \\ ${ }^{3}$ Department of Anesthesiology and Pain Medicine, College of Medicine, Inha University, Incheon, Korea
}

Supraclavicular brachial plexus blocks are not common in pediatric patients due to the risk of pneumothorax. Ultrasonography is an important tool for identifying nerves during regional anesthesia. Directly visualizing the target nerves and monitoring the distribution of the local anesthetic are potentially significant. In addition, ultrasound monitoring helps avoid complications, such as inadvertent intravascular injection or pneumothorax. This paper reports four cases of pediatric patients who received ultrasound-guided supraclavicular brachial plexus block for upper limb surgery. (Korean J Anesthesiol 2010; 59: S90-S94)

Key Words: Nerve block, Pediatrics, Ultrasonography.

Ultrasound-guided (USG) regional anesthesia and continuous perineural infusion have become increasingly popular over the last decade. The use of ultrasound (US) allows imaging of the needle, nerves, surrounding anatomical structures and spread of local anesthesia. In addition, it can help avoid complications, such as intraneural and intravascular injection [1].

Neurostimulation is the most frequently performed localizing technique for peripheral nerve blocks in pediatric patients but it cannot always be applied. US guidance can be used in pediatric patients in whom regional anesthesia would be difficult or impossible to perform [2].

This paper describes four cases, in whom USG single shot and continuous supraclavicular brachial plexus blocks were used for anesthesia and analgesia in pediatric patients.

\section{Case Reports}

\section{Case 1}

A 3-year-old boy, weighing $18 \mathrm{~kg}$, ASA physical status I, required surgery for a fracture of the lower third of the left humerus. After a thorough discussion of the risks and benefits with his parents, who then provided written informed consent, a USG supraclavicular brachial plexus block was undertaken. After inducing general anesthesia with $1 \mathrm{mg} / \mathrm{kg}$ of ketamine, a laryngeal mask airway (LMA) was inserted and ventilation was spontaneous.

A portable system with a linear array 5-10 MHz transducer (SonoSite ${ }^{\circledR} 180$ plus, Bothell, USA) was used for the USG

Received: April 26, 2010. Revised: 1st, May 14, 2010; 2nd, May 18, 2010. Accepted: May 24, 2010.

Corresponding author: Choon-Kyu Cho, M.D., Department of Anesthesiology and Pain Medicine, College of Medicine, Konyang University, 685, Gasuwon-dong, Seo-gu, Daejeon 302-718, Korea. Tel: 82-42-600-9389, Fax: 82-42-545-2132, E-mail: kl6482@naver.com

(c) This is an open-access article distributed under the terms of the Creative Commons Attribution Non-Commercial License (http:// creativecommons.org/licenses/by-nc/3.0/), which permits unrestricted non-commercial use, distribution, and reproduction in any medium, provided the original work is properly cited. 
supraclavicular brachial plexus block. The probe was placed in the coronal-oblique plane in the supraclavicular fossa and the puncture was in-plane (IP) from the lateral to medial (Fig. 1). The brachial plexus was identified as hypoechoic nodules surrounded by a hyperreflective fascial sheath, superior and lateral to the subclavian artery (Fig. 2). After infiltrating the adjacent skin and subcutaneous tissue, a 25-gauge $50-\mathrm{mm}$ insulated block needle (UniPlex NanoLine, Pajunk ${ }^{\circledR}$, Germany) was advanced along the long axis of the probe in plane with US beam guidance. When the brachial plexus bundle (Fig. 3) was reached, the nerve stimulator (Stimuplex ${ }^{\circledR}$ HNS 12, B/Braun, Germany) was turned on, starting from $1.0 \mathrm{~mA}$ and decreasing by $0.5 \mathrm{~mA}$ to elicit a muscle twitch. After injecting $1 \mathrm{ml}$ of $0.5 \%$ ropivacaine, the muscle twitch disappeared and local anesthetic spread at the time of injection was observed in real time. Nine $\mathrm{ml}$ of $0.5 \%$ ropivacaine was injected after a negative aspiration test.

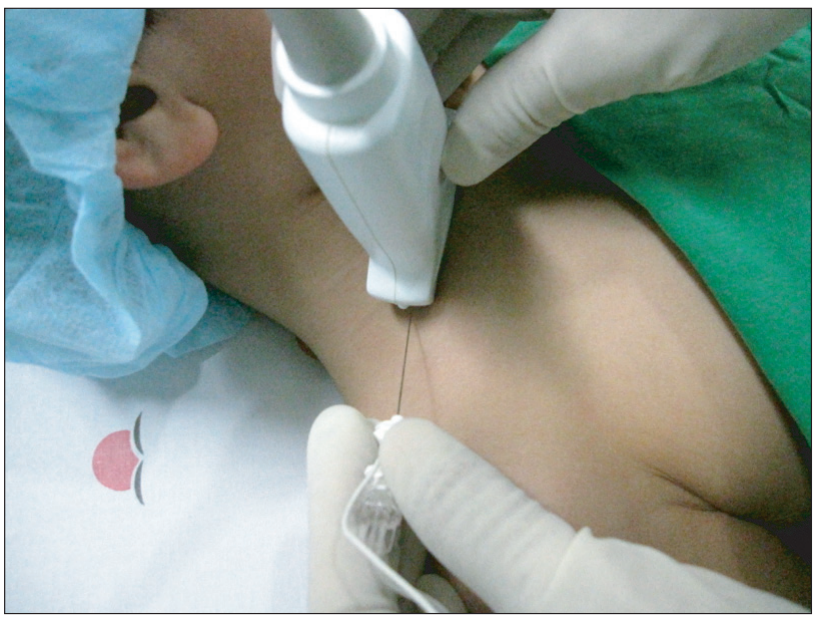

Fig. 1. In-plane technique in the supraclavicular approach.

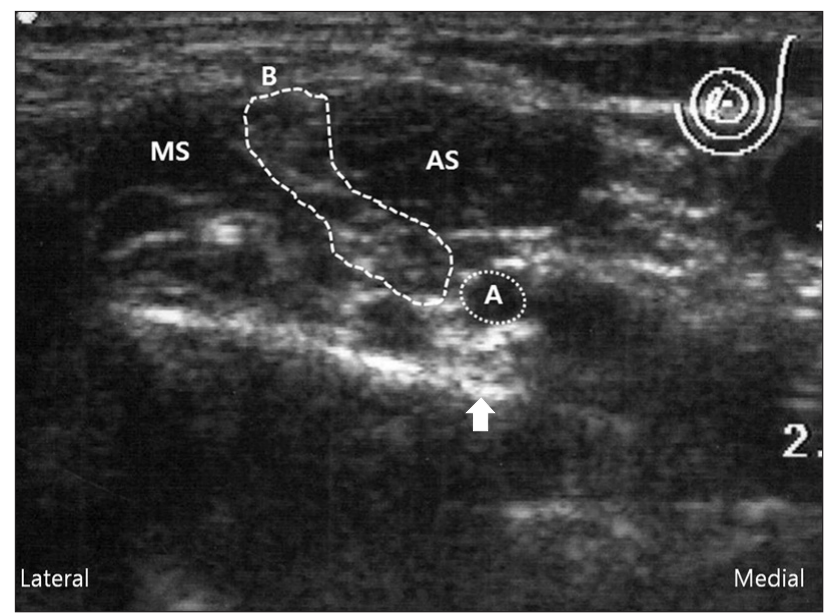

Fig. 2. Ultrasound image of the supraclavicular approach. The arrow is the first rib. A: subclavian artery, AS: anterior scalene, B: brachial plexus, MS: middle scalene; arrow.
The patient underwent a closed reduction and percutaneous pinning of the distal humerus. Maintenance of anesthesia was carried out with sevoflurane 1.5 vol\% in $50 \%$ oxygen/air until the end of the surgery. His vital signs did not change and no opioid supplementation was administered during surgery. The child did not require rescue analgesia in the postoperative period and no adverse effects were noted.

\section{Case 2}

An 11-year-old boy weighing $46 \mathrm{~kg}$, ASA physical status I, required surgery for a fracture of the upper end of the left humerus. After a thorough discussion of the risks and benefits with the patient and parents, who then provided written, informed consent, a USG supraclavicular brachial plexus block was performed.

Immediately before placing the block, midazolam $2 \mathrm{mg}$ and sufentanil $2.5 \mu \mathrm{g}$ were administered intravenously for patient comfort. A portable system with a linear array 6-13 MHz transducer (SonoSite ${ }^{\circledR}$ M-turbo, Bothell, USA) was used for the USG supraclavicular brachial plexus block. The probe was placed in the coronal-oblique plane in the supraclavicular fossa and the puncture was IP from the lateral to medial. Neurostimulation was not used to confirm the correct position. The brachial plexus was identified as hypoechoic nodules surrounded by a hyper-reflective fascial sheath, superior and lateral to the subclavian artery (Fig. 4). After infiltrating the adjacent skin and subcutaneous tissue, a 22-gauge epidural needle (Tuohy Epidural Needle ${ }^{\circledR}, \mathrm{B} /$ Braun, Germany) was advanced along the long axis of the probe in plane with the US beam. Upon reaching the brachial plexus bundle (Fig. 5), $1 \mathrm{ml}$ of $0.5 \%$ ropivacaine was injected and the local anesthetic spread at

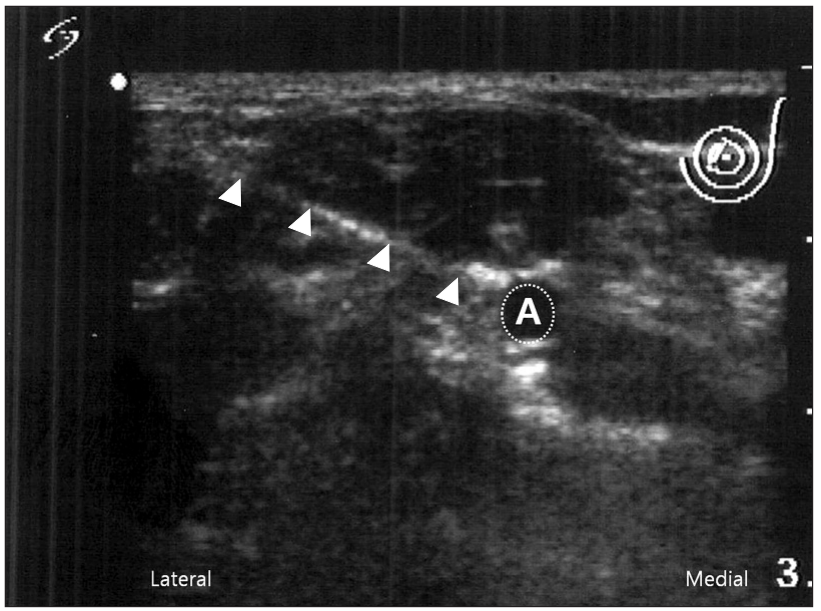

Fig. 3. Needle (arrow head) in supraclavicular approach. A: subclavian artery. 


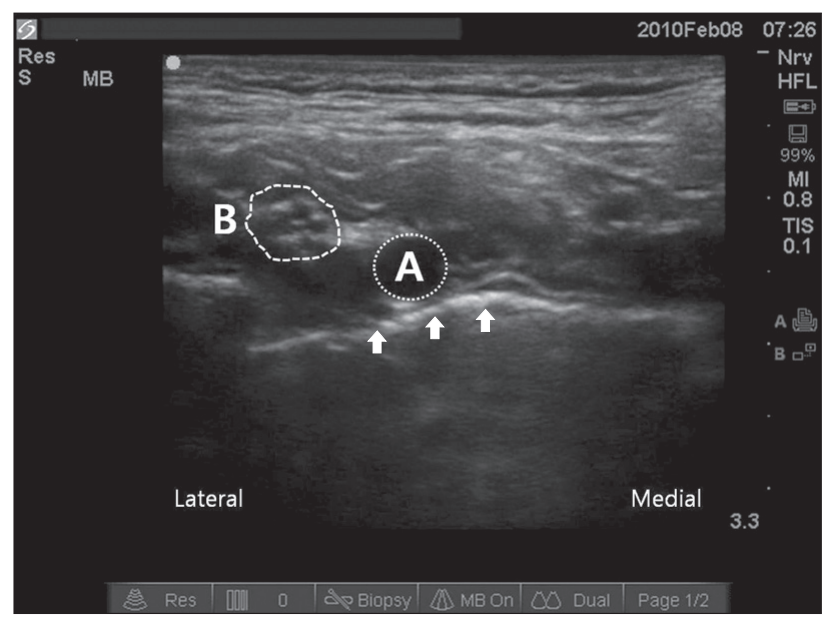

Fig. 4. Ultrasound image of the supraclavicular approach. Arrow is the first rib. A: subclavian artery, B: brachial plexus.

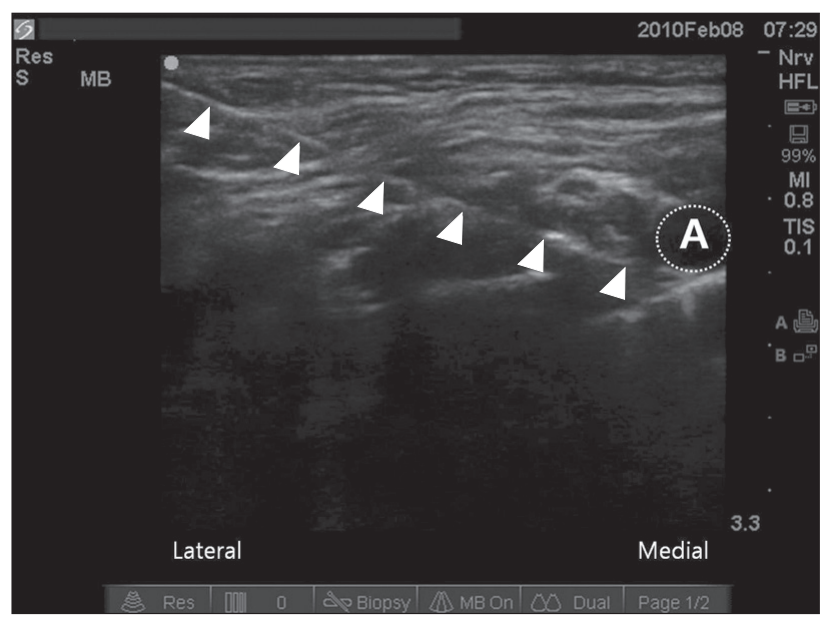

Fig. 5. Needle (arrow head) in the supraclavicular approach. A: subclavian artery.

the time of injection was observed in real time. Twenty four $\mathrm{ml}$ of $0.5 \%$ ropivacaine was injected after a negative aspiration test. Successful motor and sensory block of the upper limb occurred within $30 \mathrm{~min}$ of the injection.

The patient underwent a closed reduction and percutaneous pinning of the proximal humerus. Surgery proceeded uneventfully with no adverse effects noted.

\section{Case 3}

A 12-year-old boy weighing $45 \mathrm{~kg}$, ASA physical status I, was scheduled to undergo an open reduction and internal fixation of the radius and ulna fracture of the right forearm. After a thorough discussion of the risks and benefits with the patient and parents, who then provided written, informed consent,

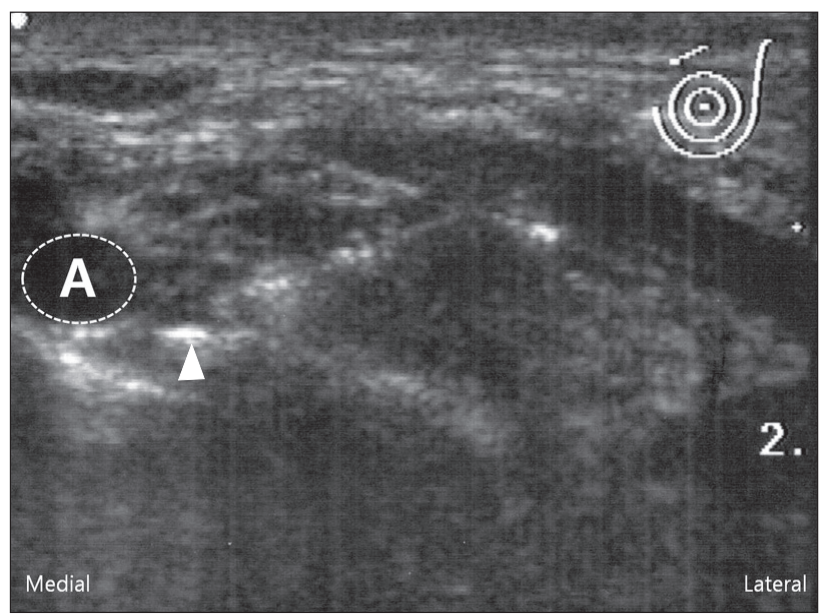

Fig. 6. Needle in the supraclavicular approach. Epidural needle tip (arrow head) was shown. A: subclavian artery.

a USG supraclavicular brachial plexus block with perineural infusion was undertaken.

Immediately before placing the block, intravenous midazolam $2 \mathrm{mg}$ and sufentanil $2.5 \mu$ g were administered. After sterile skin preparation and local anesthetic skin infiltration with $2 \%$ lidocaine, a 18 gauge Touhy-tip needle (Perican ${ }^{\circledR}, \mathrm{B} /$ Braun, Germany) was guided IP using a linear array 5-10 MHz transducer (SonoSite ${ }^{\circledR}$ 180plus, Bothell, USA) to deposit $25 \mathrm{ml}$ of $0.5 \%$ ropivacaine around the brachial plexus, which was cephalad and lateral to the subclavian artery (Fig. 6). Neurostimulation was not used to confirm the correct position. After injecting the local anesthetics, a flexible 20 gauge perineural catheter (Contiplex $\mathrm{A}^{\circledR}, \mathrm{B} / \mathrm{Braun}$, Germany) was then inserted $2 \mathrm{~cm}$ past the tip of the needle lateral to the subclavian artery. The injection port was attached to the end of the catheter, and the catheter tip position was confirmed by injecting $1 \mathrm{ml}$ of saline via the catheter under US visualization. The successful motor and sensory block of the upper limb occurred within $30 \mathrm{~min}$ of the injection.

The patient underwent an open reduction and internal fixation with a dynamic compression plate of the proximal ulna. The operation and recovery room stay were uneventful and without complications. The patient was pain free and comfortable. A $0.1 \%$ ropivacaine perineural infusion was initiated with a basal rate of $3 \mathrm{ml} / \mathrm{h}$, a patient-controlled bolus dose of $2 \mathrm{ml}$, and a lockout interval of $20 \mathrm{~min}$ via a small, light, portable electronic infusion pump (Accumate ${ }^{\circledR} 1000$, Wooyoung Medical, Korea).

The patient reported excellent postoperative analgesia without analgesic requirements during the infusion and no adverse events were encountered. The catheter was removed on the second postoperative day. 


\section{Case 4}

A 12-year-old girl weighing $47 \mathrm{~kg}$, ASA physical status I, was scheduled to undergo an open reduction and internal fixation with a plate for a Monteggia fracture of the right forearm. After a thorough discussion of the risks and benefits with the patient and parents, who then provided written, informed consent, a USG supraclavicular brachial plexus block with perineural infusion was performed.

Immediately before block placement, midazolam $2 \mathrm{mg}$ and sufentanil $2.5 \mu \mathrm{g}$ were administered intravenously. After sterile skin preparation and local anesthetic skin infiltration with $2 \%$ lidocaine, a 18 gauge Touhy-tip needle (Perican ${ }^{\circledR}, \mathrm{B} /$ Braun, Germany) was guided IP using a linear array $5-10 \mathrm{MHz}$ transducer (SonoSite ${ }^{\circledR}$ 180plus, Bothell, USA) to deposit 25 $\mathrm{ml}$ of $0.5 \%$ ropivacaine around the brachial plexus, which was cephalad and lateral to the subclavian artery. Neurostimulation was not used to confirm the correct position. After injecting local anesthetics, a flexible 20 gauge perineural catheter (Contiplex $\mathrm{A}^{\circledR}$, B/Braun, Germany) was then inserted $2 \mathrm{~cm}$ past the tip of the needle lateral to the subclavian artery. The injection port was attached to the end of the catheter, and the catheter tip position was confirmed by injecting $1 \mathrm{ml}$ of saline through the catheter under US visualization. Successful motor and sensory block of the upper limb occurred within $30 \mathrm{~min}$ of the injection.

The patient underwent an open reduction and internal fixation of the proximal ulna with a dynamic compression plate. After surgery, the patient was transported to the recovery room. The patient was pain free and comfortable. A $0.1 \%$ ropivacaine perineural infusion was initiated with a basal rate of $3 \mathrm{ml} / \mathrm{h}$, a patient-controlled bolus dose of $2 \mathrm{ml}$, and lockout interval of $20 \mathrm{~min}$ via a small, light, portable electronic infusion pump (Accumate ${ }^{\circledR} 1000$, Wooyoung Medical, Korea).

The patient only experienced pain $12 \mathrm{~h}$ after the initial injection of local anesthesia. The pain was controlled using IM diclofenac $30 \mathrm{mg}$. On the second postoperative day, she complained of an insensate extremity, which resolved spontaneously after discontinuing the local anesthetic for 2 hours. After discontinuing the infusion, she complained of pain, and a decision was made to continue the infusion. She did not complain of any pain or insensate limbs. The catheter was removed on the third postoperative day.

\section{Discussion}

The efficacy and safety of the peripheral nerve blocks (PNBs) for postoperative analgesia after orthopedic surgery has been established in adults. Recent studies have also shown earlier functional recovery after surgery in patients treated with PNBs.
PNBs in pediatric patients are emerging as a safe and effective technique for postoperative analgesia after orthopedic surgery in pediatric patients. Several studies in children have revealed the safety, feasibility, and efficacy of PNB $[3,4]$. The advantages of PNB include efficient, site-specific analgesia and a decrease in the need for opioids, as well as a decrease in the number of opioid-related side effects.

The supraclavicular brachial plexus block has an anatomical advantage of a blockade at a level where the brachial plexus elements are tightly grouped, which facilitates a single point injection and is believed to result in very rapid onset [5]. A supraclavicular brachial plexus block is ideal for surgical procedures of the hand and arm, and can also be used for shoulder surgery. However, they carry a risk of inadvertent vascular puncture or pneumothorax. Because of these issues, they are generally not recommended for routine use in pediatric patients except for those practitioners with a great deal of experience [6].

More recently, US guidance for PNBs has become a popular technique. The use of US for PNBs increases the onset time, improves the quality of the block [7] and decreases the amount of local anesthetic required [8]. However, complications, such as intravascular injection can occur even with US guidance $[9,10]$, and other routine precautions to prevent intravascular injections must be taken.

As USG regional anesthetic techniques are being used increasingly in pediatric patients, USG supraclavicular brachial plexus block has already been described in pediatric patients [11]. The main advantages of US guidance in the supraclavicular approach are visualization of the surrounding structures (pleura, subclavian artery and vein) and the real-time control of needle movement, both of which reduce the risk of pneumothorax.

Generally, there are two techniques for orienting the needle with respect to the US beam. The IP view generates a long axis view of the needle, allowing full visualization of the shaft and tip of the needle. The out-of-plane view generates a short axis view of the needle. We decided to use the IP technique because it is the only possible approach to the supraclavicular brachial plexus that decreases the risk of pleural puncture. In addition, the brachial plexus in the supraclavicular fossa is superficial and the space to introduce the needle IP from the lateral to medial is usually sufficient, even in pediatric patients (elevation of the shoulders might be necessary in very small pediatric patients). A hockey stick linear probe would certainly be preferable.

In this report, USG perineural catheter placement was performed. Continuous perineural infusion provided excellent postoperative analgesia with no adverse events. Continuous peripheral nerve blocks (CPNB) provide pain relief that is specific to the site of surgery with minimal side effects for hospitalized and ambulatory pediatric patients $[4,12]$. However, 
CPNB was not compared with other methods of postoperative analgesia. Therefore, it is impossible to demonstrate the superiority of CPNB.

Epinephrine prolongs the duration and intensity of most local anesthetics used for PNB and acts as a marker of intravascular injection and potentially limiting systemic local anesthetic toxicity by reducing the time-to-peak concentration and peak plasma concentration, even though the latter effect was not observed with ropivacaine [13]. Epinephrine-containing local anesthetics were not used. First, the addition of epinephrine does not alter the pharmacokinetic properties of ropivacaine when used for a brachial plexus block. Second, US can allow visualization of local anesthetic spread around the neural target and helps avoid complications, such as inadvertent intravascular injection. However, the use of US per se does not eliminate the intravascular injection of local anesthetic [9]. Third, epinephrine is likely to be safe when applied to the nerve fascicles with intact tissue barriers but may accentuate the injury in the event of barrier disruption, which can occur after an intraneural injection [14] or in individuals with chemotherapy-related neurotoxicity, diabetic neuropathies [15] or atherosclerosis.

In summary, US localization of the nerves is a useful adjunct for applications of PNBs in pediatric patients. Although there is some debate as to whether US is actually beneficial in regional anesthesia, this report demonstrates the feasibility of USG supraclavicular brachial plexus blocks in pediatric patients. However, more experience with this technique is needed.

\section{References}

1. Marhofer P, Greher M, Kapral S. Ultrasound guidance in regional anaesthesia. Br J Anaesth 2005; 94: 7-17.

2. Jan van Geffen G, Tielens L, Gielen M. Ultrasound-guided interscalene brachial plexus block in a child with femur fibula ulna syndrome. Paediatr Anaesth 2006; 16: 330-2.

3. Dadure C, Capdevila X. Continuous peripheral nerve blocks in children. Best Pract Res Clin Anaesthesiol 2005; 19: 309-21.
4. Ganesh A, Rose JB, Wells L, Ganley T, Gurnaney H, Maxwell LG, et al. Continuous peripheral nerve blockade for inpatient and outpatient postoperative analgesia in children. Anesth Analg 2007; 105: 1234-42.

5. Franco CD, Vieira ZE. 1,001 subclavian perivascular brachial plexus blocks: success with a nerve stimulator. Reg Anesth Pain Med 2000; 25: 41-6.

6. Dalens B. Regional anesthesia in children. Anesth Analg 1989; 68: 654-72.

7. Marhofer P, Schrogendorfer K, Koinig H, Kapral S, Weinstabl C, Mayer N. Ultrasonographic guidance improves sensory block and onset time of three-in-one blocks. Anesth Analg 1997; 85: 854-7.

8. Marhofer P, Schrogendorfer K, Wallner T, Koinig H, Mayer N, Kapral S. Ultrasonographic guidance reduces the amount of local anesthetic for 3-in-1 blocks. Reg Anesth Pain Med 1998; 23: 584-8.

9. Zetlaoui PJ, Labbe JP, Benhamou D. Ultrasound guidance for axillary plexus block does not prevent intravascular injection. Anesthesiology 2008; 108: 761.

10. Loubert C, Williams SR, Helie F, Arcand G. Complication during ultrasound-guided regional block: accidental intravascular injection of local anesthetic. Anesthesiology 2008; 108: 759-60.

11. De Jose Maria B, Banus E, Navarro Egea M, Serrano S, Perello M, Mabrok M. Ultrasound-guided supraclavicular vs infraclavicular brachial plexus blocks in children. Paediatr Anaesth 2008; 18: 83844.

12. Dadure C, Pirat P, Raux O, Troncin R, Rochette A, Ricard C, et al. Perioperative continuous peripheral nerve blocks with disposable infusion pumps in children: a prospective descriptive study. Anesth Analg 2003; 97: 687-90.

13. Hickey R, Blanchard J, Hoffman J, Sjovall J, Ramamurthy S. Plasma concentrations of ropivacaine given with or without epinephrine for brachial plexus block. Can J Anaesth 1990; 37: 878-82.

14. Selander D, Brattsand R, Lundborg G, Nordborg C, Olsson Y. Local anesthetics: importance of mode of application, concentration and adrenaline for the appearance of nerve lesions. An experimental study of axonal degeneration and barrier damage after intrafascicular injection or topical application of bupivacaine (Marcain). Acta Anaesthesiol Scand 1979; 23: 127-36.

15. Schneider U, Jund R, Nees S, Grafe P. Differences in sensitivity to hyperglycemic hypoxia of isolated rat sensory and motor nerve fibers. Ann Neurol 1992; 31: 605-10. 